\title{
Simultaneous Tridiagonalization of Two Symmetric Matrices
}

\author{
Seamus. D. Garvey ${ }^{1}$, Françoise Tisseur ${ }^{2}$, \\ Michael I. Friswell ${ }^{3}$, John E. T. Penny ${ }^{4}$, Uwe Prells ${ }^{3}$ \\ 1 School of Mechanical, Materials, Manufacturing Engineering 83 Management, \\ University of Nottingham, University Park, Nottingham NG7 2RD. \\ 2 Department of Mathematics, University of Manchester, Manchester, M13 9PL. \\ 3 University of Wales, Swansea Singleton Park, Swansea, SA2 8PP. \\ 4 University of Aston, Birmingham, B4 $7 E T$.
}

\begin{abstract}
SUMMARY
We show how to simultaneously reduce a pair of symmetric matrices to tridiagonal form by congruence transformations. No assumptions are made on the nonsingularity or definiteness of the two matrices. The reduction follows a strategy similar to the one used for the tridiagonalization of a single symmetric matrix via Householder reflectors. Two algorithms are proposed, one using non-orthogonal rank-one modifications of the identity matrix and the other, more costly but more stable, using a combination of Householder reflectors and non-orthogonal rank-one modifications of the identity matrix with minimal condition numbers. Each of these tridiagonalization processes requires $O\left(n^{3}\right)$ arithmetic operations and respects the symmetry of the problem. We illustrate and compare the two algorithms with some numerical experiments. Copyright (c) 2002 John Wiley \& Sons, Ltd.
\end{abstract}

KEY WORDS: Symmetric matrices, generalized eigenvalue problem, tridiagonalization, symmetric quadratic eigenvalue problem

\section{INTRODUCTION}

This paper concerns pairs of symmetric matrices $(K, M)$ and the computation of a nonsingular transformation $Q$ that simultaneously tridiagonalizes the pair $(K, M)$, that is,

$$
Q^{T} K Q=T, \quad Q^{T} M Q=S,
$$

where both $T$ and $S$ are symmetric tridiagonal. No assumptions are made on the nonsingularity or definiteness of $M$ and $K$.

${ }^{*}$ Correspondence to: Seamus. D. Garvey, School of Mechanical, Materials, Manuf. Eng. \& Management, University of Nottingham, University Park, Nottingham NG7 2RD.

Contract/grant sponsor: EPSRC; contract/grant number: GR/M93062, GR/M93079, GR/R45079/01

Contract/grant sponsor: Nuffield Foundation; contract/grant number: NAL/00216/G 


\subsection{Motivation}

Our main motivation for reducing a symmetric pair $(K, M)$ to symmetric tridiagonal form $(T, S)$ arises from the modeling of undamped multi-degree of freedom second-order systems

$$
\begin{aligned}
& K q+M \ddot{q}=B f, \\
& r=B^{T} q,
\end{aligned}
$$

and multi-degree of freedom first-order systems

$$
\begin{aligned}
& K q+D \dot{q}=B f, \\
& r=B^{T} q,
\end{aligned}
$$

where $q$ is the vector of displacements and $r$ and $f$ are the vectors of terminal displacements and forces, $r$ and $f$ generally being of much smaller dimension than the displacement $q$. The matrix $B$ is a selection matrix relating the full-length vectors to the corresponding terminal quantities. Three types of analysis are commonly performed for systems such as (2) and (3).

1. Computation of the transient response $q$ as a function of time $t$.

2. Computation of the steady-state frequency response $q$ as a function of the frequency $\omega$.

3. Computation of the natural frequencies $\omega_{n}$.

For the reminder of this section, these analyses will be discussed as though they pertained only to the system of (2). Equivalent statements apply in all cases to systems such as (3).

As long as we can determine the acceleration $\ddot{q}$ at any instant $t$, the transient responses may be computed by conducting a time-marching integration. Note that

$$
\ddot{q}(t)=M^{-1}(B f(t)-K q(t)),
$$

so that on each occasion where $\ddot{q}$ is determined, it is necessary to perform one matrix-vector multiplication with $K$ and solve a system of equations with $M$. If $M^{-1}$ is computed initially and then stored, the computation of each new $\ddot{q}$ requires $O\left(n^{2}\right)$ operations, where $n$ is the dimension of the problem. Assume that $(K, M)$ has been reduced to tridiagonal form $(T, S)$ via the transformation $Q$ and let $p$ and $C$ be defined by $q=Q p$ and $C=Q^{T} B$. Then (2) becomes

$$
\begin{aligned}
& T p+S \ddot{p}=C f, \\
& r=C^{T} p .
\end{aligned}
$$

With this equivalent representation of the system, each computation of $\ddot{p}$ requires a matrixvector product and the solution of a linear system with a tridiagonal matrix. Both operations can be done $O(n)$ operations.

The advantage afforded by the reduction to tridiagonal form (1) in the computation of steady-state frequency response is even more striking. To obtain the steady-state frequency response of the original system (2) directly as a function of $\omega$ we have to compute

$$
r(\omega)=B^{T}\left(K-\omega^{2} M\right)^{-1} B f(\omega) .
$$

This involves the solution of a system of coupled equations at each distinct frequency and the associated computational burden is $O\left(n^{3}\right)$ operations. This burden is reduced to $O\left(n^{2}\right)$ when the tridiagonalizing transformation is applied so that $(T, S)$ appears in place of $(K, M)$.

Another motivation for this work is that the first step in most natural frequency or eigenvalue computations is the reduction, in a finite number of operations, to a simple form such as the tridiagonal reduction (1). Then an iterative procedure can be applied to compute the eigensystem efficiently. 


\subsection{Review of existing reductions}

To put our work into perspective, we review some of the existing methods for reducing, in a finite number of steps, a pair of symmetric matrices $(K, M)$ to some simple form and give the conditions that must be satisfied by $K$ and $M$ for the reduction to be possible. We concentrate on methods that preserve symmetry. All the methods we are aware of reduce $K$ to tridiagonal form and $M$ to diagonal form.

Generally, when $M$ is positive definite $(M>0)$, a Cholesky factorization of $M=L L^{T}$ is used to transform $(K, M)$ into $(A, I)$ with $A=L^{-1} K L^{-T}$ symmetric. Then $A$ is tridiagonalized into $T$ via a sequence of Householder transformations [5]. It is well-known that when $M$ is close to being singular, the computation of $A$ may suffer from instability.

When $M$ is positive semidefinite $(M \geq 0)$, with $r>0$ zero eigenvalues, we can again transform $(K, M)$ to tridiagonal-diagonal form $(A, D)$ with $D=\left[\begin{array}{cc}I_{n-r} & 0 \\ 0 & 0_{r}\end{array}\right]$, but the reduction is not as straightforward as in the previous case. It can be done by a Cholesky factorization for semidefinite matrices followed by one step of Bunse-Gerstner's MDR reduction for symmetric matrices [2] (to split the problem into two subproblems, one of them corresponding to the $r$ zero eigenvalues), and then a judicially chosen sequence of Givens rotations.

If $M$ is indefinite, that is, $M$ has both positive and negative eigenvalues, $(K, M)$ can be reduced to tridiagonal-diagonal form using one of the procedures described by Brebner and Grad [1] or by Zurmühl and Falk [10]. However, these reductions require $M$ to be nonsingular.

\subsection{Objectives}

The symmetric tridiagonal-diagonal reduction is the most compact form we can obtain in a finite number of steps and the price to pay for this may be numerical instability. In this paper we consider a less compact form that allows the second matrix to be in tridiagonal form.

One feature of our algorithm not shared by any other algorithm reducing a pair of symmetric matrices to some simple symmetric form is that it is not necessary that either of the two matrices should be positive definite or even positive semidefinite. Although it is common that in structural vibration and other undamped second order dynamic systems both matrices are at least positive semidefinite, the extension to damped second order systems

$$
M \ddot{q}+C \dot{q}+K q=f,
$$

where $C=C^{T}$ is the damping matrix (often positive semidefinite), leads to first order systems

$$
\mathcal{K} p+\mathcal{M} \dot{p}=g, \quad p=\left[\begin{array}{l}
q \\
\dot{q}
\end{array}\right], \quad g=\left[\begin{array}{l}
0 \\
f
\end{array}\right],
$$

where $\mathcal{K}$ and $\mathcal{M}$ are symmetric matrices neither of which is positive definite [3], [9]. Hence our tridiagonal reduction works in the most general case and can be used when nothing is known about the pair $(K, M)$ other than its symmetry.

The paper is organized as follows. We describe in section 2 the simultaneous tridiagonalization of a symmetric pair $(K, M)$ and define the transformations required at each step of the reduction. We explain in section 3 how to compute these transformations efficiently. We present Algorithm 3.1 which is based on $n-2$ successive transformations, each one being a non-orthogonal rank-one modification of the identity matrix. We show in section 4 that in order to minimize the growth of inherent errors in the pair $(K, M)$ it is crucial to keep the condition 
number of the transformations used during the tridiagonalization process small. We describe a new type of elementary transformation based on the product of a Householder reflector and a non-orthogonal rank-one modification of the identity matrix with minimal condition number. We use these new transformations to derive Algorithm 4.2. Both algorithms depend on a parameter $\gamma$. We give in section 5 some heuristics for choosing this parameter. Section 6 is devoted to numerical experiments.

\subsection{Notation}

Generally we use capital letters for matrices, lower case letters for vectors and lower Greek letters for scalars. $I$ is the identity matrix whose dimension is determined by the context, and the vector $e_{k}$ denotes its $k$ th column. We often use the rectangular matrix

$$
V=\left[e_{2}, e_{3}, \ldots, e_{\ell}\right] \in \mathbb{R}^{\ell \times(\ell-1)} .
$$

Premultiplication of an $\ell \times \ell$ matrix by $V^{T}$ discards the first row of the matrix. We denote by

$$
\kappa(A)=\|A\|\left\|A^{-1}\right\|
$$

the condition number of a square matrix $A,\|\cdot\|$ being the 2-norm (Euclidean norm). Finally the colon notation $i=1: n$ means the same as $i=1, \ldots, n$.

\section{REDUCTION TO TRIDIAGONAL FORMS}

In this section we describe our technique for reducing a pair of symmetric $n \times n$ matrices $(K, M)$ to tridiagonal form $(T, S)$. Congruence transformations are used to preserve symmetry and eigenvalues.

\subsection{Basic idea}

Assume that there exists a nonsingular $n \times n$ matrix $G_{1}$ that introduces zeros in the first column below the first subdiagonal and in the first row after the first superdiagonal of both $K_{1} \equiv K$ and $M_{1} \equiv M$. This gives

$$
K_{2}=G_{1}^{T} K_{1} G_{1}=\left[\begin{array}{cc}
\kappa_{1} & \tau_{1} e_{1}^{T} \\
\tau_{1} e_{1} & \widetilde{K}_{2}
\end{array}\right], \quad M_{2}=G_{1}^{T} M_{1} G_{1}=\left[\begin{array}{cc}
\mu_{1} & \sigma_{1} e_{1}^{T} \\
\sigma_{1} e_{1} & \widetilde{M}_{2}
\end{array}\right]
$$

where $\kappa_{1}, \tau_{1}, \mu_{1}$ and $\sigma_{1}$ are real scalars. This causes $K_{2}$ and $M_{2}$ to be tridiagonal in their first rows and columns.

At the second step, the same idea is applied to $\widetilde{K}_{2}$ and $\widetilde{M}_{2}$. We denote by $\widetilde{G}_{2}$ the nonsingular matrix introducing zeros in the first column and first row of both $\widetilde{K}_{2}$ and $\widetilde{M}_{2}$,

$$
\widetilde{G}_{2}^{T} \widetilde{K}_{2} \widetilde{G}_{2}=\left[\begin{array}{cc}
\kappa_{2} & \tau_{2} e_{1}^{T} \\
\tau_{2} e_{1} & \widetilde{K}_{3}
\end{array}\right], \quad \widetilde{G}_{2}^{T} \widetilde{M}_{2} \widetilde{G}_{2}=\left[\begin{array}{cc}
\mu_{2} & \sigma_{2} e_{1}^{T} \\
\sigma_{2} e_{1} & \widetilde{M}_{3}
\end{array}\right],
$$

and let $G_{2}=\left[\begin{array}{cc}1 & 0 \\ 0 & \widetilde{G}_{2}\end{array}\right]$. Note that for the two matrices

$$
K_{3}=G_{2}^{T} K_{2} G_{2}, \quad M_{3}=G_{2}^{T} M_{2} G_{2}
$$


to be tridiagonal in their two first columns, it is crucial that $G_{2}$ does not destroy the zeros already introduced in the first columns and rows of $K_{2}$ and $M_{2}$. As a consequence, $\widetilde{G}_{2}$ must satisfy

$$
\widetilde{G}_{2}^{T} e_{1}=\beta e_{1}, \quad 0 \neq \beta \in \mathbb{R} .
$$

Hence

$$
K_{3}=\left[\begin{array}{ccc}
\kappa_{1} & \beta \tau_{1} & 0^{T} \\
\beta \tau_{1} & \kappa_{2} & \tau_{2} e_{1}^{T} \\
0 & \tau_{2} e_{1} & \widetilde{K}_{3}
\end{array}\right], \quad M_{3}=\left[\begin{array}{ccc}
\mu_{1} & \beta \sigma_{1} & 0^{T} \\
\beta \sigma_{1} & \mu_{2} & \sigma_{2} e_{1}^{T} \\
0 & \sigma_{2} e_{1} & \widetilde{M}_{3}
\end{array}\right]
$$

Finally, after $n-2$ such steps,

$$
K_{n-1}=G_{n-2}^{T} K_{n-2} G_{n-2} \equiv T, \quad M_{n-1}=G_{n-2}^{T} M_{n-2} G_{n-2} \equiv S
$$

are in tridiagonal form. Let

$$
Q=G_{1} G_{2} \ldots G_{n-2}
$$

Then $Q$ simultaneously tridiagonalizes $K$ and $M$,

$$
T=Q^{T} K Q, \quad S=Q^{T} M Q .
$$

In what follows we describe how to construct the matrices $G_{k}, k=1: n-2$. As the technique is the same at each step $k$ of the reduction, we drop the subscript $k$.

\subsection{Constructing $G$}

We examine how to construct a nonsingular matrix $G \in \mathbb{R}^{\ell \times \ell}$ satisfying

$$
G^{T} e_{1}=e_{1}, \quad G^{T} K G=\left[\begin{array}{cc}
\kappa & \tau e_{1}^{T} \\
\tau e_{1} & \widetilde{K}
\end{array}\right], \quad G^{T} M G=\left[\begin{array}{cc}
\mu & \sigma e_{1}^{T} \\
\sigma e_{1} & \widetilde{M}
\end{array}\right],
$$

where $\kappa, \mu, \sigma, \tau$ are real scalars and, $K, M$ are symmetric matrices. The first constraint in (7) comes from (6), where without loss of generality we set $\beta=1$.

We recall that the role of $G$ is to introduce zeros in the first column below the first superdiagonal and in the first row after the first superdiagonal of both $K$ and $M$. Here the $\ell \times \ell$ matrices $G, K$ and $M$ can be seen as the matrices $G_{k}, \widetilde{K}_{k}$ and $\widetilde{M}_{k}$ in section 2.1 with $\ell=n-k+1$.

An $\ell \times \ell$ Householder reflector has the form

$$
H=I-2 \frac{v v^{T}}{v^{T} v}, \quad 0 \neq v \in \mathbb{R}^{\ell} .
$$

Householder reflectors are a powerful tool for introducing zeros into vectors. For any $u \in \mathbb{R}^{\ell}$, if

$$
v=u+\operatorname{sign}\left(e_{1}^{T} u\right)\|u\| e_{1},
$$

then

$$
H u=-\operatorname{sign}\left(e_{1}^{T} u\right)\|u\| e_{1} .
$$

Householder reflectors are symmetric, orthogonal and they enjoy good numerical properties (see [7, ch. 19]). We denote by

$$
v=\operatorname{house}(u)
$$

any function (program) computing $v$ so that (8) is satisfied.

Two situations are considered for the construction of $G$. 
(i) If $V^{T} K e_{1}=\alpha V^{T} M e_{1}$ for some $\alpha \in \mathbb{R}$ with $V$ as in (5) then taking $L=I$ and constructing a Householder matrix $H$ such that

$$
H^{T} V^{T} K e_{1}=\tau e_{1}
$$

yields $H^{T} V^{T} M e_{1}=\alpha \tau e_{1}$. Then

$$
G=\operatorname{diag}(1, H)
$$

satisfies (7).

(ii) Assume that $V^{T} K e_{1} \neq \alpha V^{T} M e_{1}$ for all $\alpha \in \mathbb{R}$, that is $V^{T} K e_{1}$ and $V^{T} M e_{1}$ are linearly independent. If $K$ and $M$ can be transformed by congruence transformation with a nonsingular matrix $L \neq I$ in a such way that

$$
V^{T} L^{T} K L e_{1}=\alpha V^{T} L^{T} M L e_{1}
$$

for some $\alpha \in \mathbb{R}$, then we are back in case (i) with $K$ and $M$ replaced by $L^{T} K L$ and $L^{T} M L$ respectively. The role of $L$ is to transform the first columns and rows of $K$ and $M$ so that a Householder reflector can subsequently create the requisite zeros simultaneously in both matrices. Hence if $L^{T} e_{1}=e_{1}$ then

$$
G=L\left[\begin{array}{cc}
1 & 0 \\
0 & H
\end{array}\right]
$$

satisfies (7). Note that if there exists an $L$ such that the two vectors $V^{T} L^{T} K L e_{1}$ and $V^{T} L^{T} M L e_{1}$ have zeros in all their components except the first then we can set $H=I$.

In the next section we show how to construct the matrix $L$ of case (ii).

\subsection{Constructing $L$}

We describe a class of nonsingular elementary matrices $L \neq I \in \mathbb{R}^{\ell \times \ell}$ of the form

$$
L=I+x y^{T}, \quad x, y \in \mathbb{R}^{\ell}, \quad x^{T} y \neq-1,
$$

such that

$$
e_{1}^{T} L=e_{1}^{T}, \quad V^{T}\left(L^{T} K L\right) e_{1}=\widetilde{\kappa} w, \quad V^{T}\left(L^{T} M L\right) e_{1}=\widetilde{\mu} w,
$$

with $w \in \mathbb{R}^{\ell-1}, \widetilde{\kappa}, \widetilde{\mu}$ nonzero scalars, and with the assumption that the two vectors $V^{T} K e_{1}$ and $V^{T} M e_{1}$ are linearly independent. If $V^{T} K e_{1}$ and $V^{T} M e_{1}$ are linearly dependent then we can take $L=I$. The condition $x^{T} y \neq-1$ ensures that $L$ is nonsingular.

The matrices $L$ are rank-one modifications of the identity matrix. They have interesting mathematical and numerical properties. In particular their inverse is explicitly given by

$$
L^{-1}=I-\frac{x y^{T}}{1+x^{T} y}
$$

and their condition number is readily available in terms of $x$ and $y$ (see section 4). Also the transformation $L^{T} A L$ with $A$ symmetric can be done in $4 \ell^{2}$ operations. This can be expressed in MATLAB-style pseudocode as follows. 
function $A=\operatorname{elm} \_\operatorname{apply}(A, x, y)$

$\%$ Apply elementary transformation $L=I+x y^{T}$ to $A$.

$z=A x$

$u=z+\left(x^{T} z\right) y / 2$

$A=A+u y^{T}+y u^{T}$

In the rest of this section we concentrate on deriving the vectors $x$ and $y$ defining $L$. The first constraint in (11) implies that

$$
e_{1}^{T} x=0
$$

Let us assume that $e_{1}^{T} y=0$. Then

$$
L=\left[\begin{array}{cc}
1 & 0 \\
0 & \widetilde{L}
\end{array}\right], \quad \widetilde{L}=I-\widetilde{x} \widetilde{y}^{T}, \quad \widetilde{x} \widetilde{y} \in \mathbb{R}^{\ell-1}
$$

and the two last constraints in (11) become

$$
V^{T}\left[\left(L^{T} K L\right) e_{1} \quad\left(L^{T} K L\right) e_{1}\right]=\widetilde{L}\left[\begin{array}{ll}
V^{T} K e_{1} & V^{T} M e_{1}
\end{array}\right]=\left[\begin{array}{ll}
\widetilde{\kappa} w & \widetilde{\mu} w
\end{array}\right]
$$

As $V^{T} K e_{1}$ and $V^{T} M e_{1}$ are linearly independent, $\widetilde{L}$ transforms a rank-2 matrix into a rank-1 matrix, implying that $\widetilde{L}$ and therefore also $L$ is singular. So $e_{1}^{T} y \neq 0$ and without loss of generality we take

$$
e_{1}^{T} y=1 .
$$

The next two constraints in (11) can be rewritten as

$$
\begin{aligned}
V^{T} K\left(x+e_{1}\right)+\left[\left(x+e_{1}\right)^{T} K x\right] V^{T} y & =\widetilde{\kappa} w, \\
V^{T} M\left(x+e_{1}\right)+\left[\left(x+e_{1}\right)^{T} M x\right] V^{T} y & =\widetilde{\mu} w .
\end{aligned}
$$

Let $\gamma$ be a fixed nonzero constant such that $K-\gamma M$ is nonsingular. We now look for a solution $x$ that satisfies the simplifying condition

$$
\left(x+e_{1}\right)^{T} K x=\gamma\left(x+e_{1}\right)^{T} M x .
$$

We also impose that the scalars $\widetilde{\kappa}, \widetilde{\mu}$ in (11) satisfy

$$
\widetilde{\kappa}=\gamma \widetilde{\mu} .
$$

Then, (14) $-\gamma \times(15)$ yields the underdetermined system

$$
V^{T}(K-\gamma M)\left(x+e_{1}\right)=0,
$$

whose solutions are given by

$$
(K-\gamma M)\left(x+e_{1}\right)=\delta_{1} e_{1}, \quad \delta_{1} \in \mathbb{R} \text { arbitrary } .
$$

Since $K-\gamma M$ is nonsingular, we have

$$
x+e_{1}=\delta_{1}(K-\gamma M)^{-1} e_{1} .
$$

The condition $e_{1}^{T} x=0$ in (12) implies

$$
\delta_{1}=\left(e_{1}^{T}(K-\gamma M)^{-1} e_{1}\right)^{-1} .
$$


Hence

$$
x=\left(e_{1}^{T}(K-\gamma M)^{-1} e_{1}\right)^{-1}(K-\gamma M)^{-1} e_{1}-e_{1} .
$$

It is easy to verify that with this expression for $x$, the simplifying condition in (16) is satisfied.

We now determine the vector $y$. Forming $\gamma \times(14)+(15)$ gives

$$
V^{T}(\gamma K+M)\left(x+e_{1}\right)+\left(\gamma\left(x+e_{1}\right)^{T} K x+\left(x+e_{1}\right)^{T} M x\right) V^{T} y=(\gamma \widetilde{\kappa}+\widetilde{\mu}) w .
$$

Note that because of (17), $\widetilde{\kappa}, \widetilde{\mu}$ are determined up to a constant. We now fix them completely with the normalization condition

$$
\gamma \widetilde{\kappa}+\widetilde{\mu}=\left(x+e_{1}\right)^{T}(\gamma K+M) x .
$$

Hence

$$
V^{T} y=-\left[\left(x+e_{1}\right)^{T}(\gamma K+M) x\right]^{-1} V^{T}(\gamma K+M)\left(x+e_{1}\right)+w .
$$

All the solutions to this underdetermined system are given by

$$
y=-\left[\left(x+e_{1}\right)^{T}(\gamma K+M) x\right]^{-1} V V^{T}(\gamma K+M)\left(x+e_{1}\right)+V w+\delta_{2} e_{1},
$$

for some arbitrary $\delta_{2} \in \mathbb{R}$. The condition $e_{1}^{T} y=1$ in (13) yields $\delta_{2}=1$.

To summarize, given an arbitrary vector $w \in \mathbb{R}^{\ell-1}$ and a nonzero scalar $\gamma$ such that $K-\gamma M$ is nonsingular, the matrix $L=I+x y^{T}$ with

$$
\begin{aligned}
x & =\left(e_{1}^{T}(K-\gamma M)^{-1} e_{1}\right)^{-1}(K-\gamma M)^{-1} e_{1}-e_{1}, \\
y & =-\left[\left(x+e_{1}\right)^{T}(\gamma K+M) x\right]^{-1} V V^{T}(\gamma K+M)\left(x+e_{1}\right)+V w+e_{1}
\end{aligned}
$$

satisfies

$$
e_{1}^{T} L=e_{1}^{T}, \quad V^{T}\left(L^{T} K L\right) e_{1}=\widetilde{\kappa} w, \quad V^{T}\left(L^{T} M L\right) e_{1}=\widetilde{\mu} w .
$$

Note that $L$ is nonsingular for $w$ such that $x^{T} y \neq-1$.

Assume that $w$ and $z=(K-\gamma M)^{-1} e_{1}$ are given. The computation of $x$ and $y$ can be written in pseudocode as follows:

$$
\begin{aligned}
& \text { function }[x, y]=\operatorname{elm}_{-} \mathrm{w}_{-} \mathrm{xy}(K, M, \gamma, w, z) \\
& \% \text { For } w \text { and } z=(K-\gamma M)^{-1} e_{1} \text { given, construct the vectors } x, y \in \mathbb{R}^{\ell} \\
& \text { \% so that } L=I+x y^{T} \text { satisfies }(11) . \\
& k=K(2: \ell, 1), m=M(2: \ell, 1) \\
& \text { If }\left|k^{T} m\right|=\|k\|_{2}\|m\|_{2} \\
& \quad x=0, y=0 \\
& \text { else } \\
& \qquad \begin{array}{l}
x=z / z(1) \\
u=(\gamma K+M) x, x(1)=0 \\
y=-u /\left(u^{T} x\right) \\
\text { end }
\end{array}
\end{aligned}
$$

The first test checks to see whether $k$ and $m$ are linearly dependent. This algorithm requires about $2 \ell^{2}$ operations. 


\section{COMPUTING THE TRANSFORMATIONS $G_{k}$ EFFICIENTLY}

We now return to the notation used in section 2.1. Recall that at step $k$ of the reduction to tridiagonal forms, we have to compute the vectors $x_{k}, y_{k}$ and $v_{k}$ defining the the $n \times n$ transformation $G_{k}$,

$$
G_{k}=\left[\begin{array}{cc}
I & 0 \\
0 & \widetilde{G}_{k}
\end{array}\right], \quad \widetilde{G}_{k}=L_{k}\left[\begin{array}{cc}
1 & 0 \\
0 & H_{k}
\end{array}\right] \in \mathbb{R}^{(n-k+1) \times(n-k+1)} .
$$

The main numerical difficulty is in the computation of

$$
x_{k}=\left(e_{1}^{T}\left(\widetilde{K}_{k}-\gamma \widetilde{M}_{k}\right)^{-1} e_{1}\right)^{-1}\left(\widetilde{K}_{k}-\gamma \widetilde{M}_{k}\right)^{-1} e_{1}-e_{1} .
$$

Forming all the $x_{k} \in \mathbb{R}^{n-k+1}, k=1: n-2$ requires the solution of $n-2$ symmetric, possibly indefinite, systems of $n-k$ equations,

$$
\left(\widetilde{K}_{k}-\gamma \widetilde{M}_{k}\right) z_{k}=e_{1}, \quad k=1: n-2,
$$

and the whole tridiagonalization procedure requires $O\left(n^{4}\right)$ operations if the systems $(24)$ are solved as arbitrary systems. We describe in this section a way of solving the $n-2$ systems $(24)$ in $O\left(n^{3}\right)$ operations.

At step $k$ of the tridiagonalization procedure we have

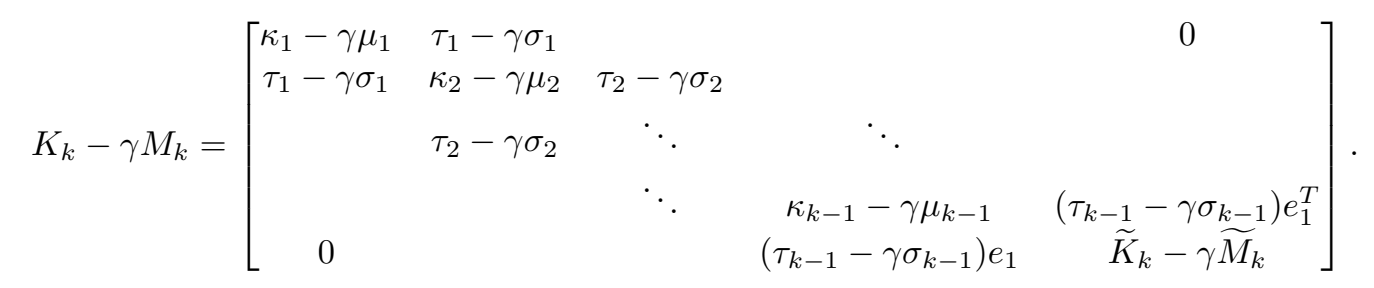

By construction,

$$
\tau_{j}=-\widetilde{\kappa}_{j} \operatorname{sign}\left(w_{j}(1)\right)\left\|w_{j}\right\|, \quad \sigma_{j}=-\widetilde{\mu}_{j} \operatorname{sign}\left(w_{j}(1)\right)\left\|w_{j}\right\|, \quad j=1: k-1,
$$

with $\widetilde{\kappa}_{j}, \widetilde{\mu}_{j}$ satisfying (17), that is, $\widetilde{\kappa}_{j}=\gamma \widetilde{\mu}_{j}$. This gives

$$
\tau_{j}-\gamma \sigma_{j}=0, \quad j=1: k-1 .
$$

Hence at the $k$ th step the matrix $K_{k}-\gamma M_{k}$ is diagonal in its first $k$ rows and columns,

$$
K_{k}-\gamma M_{k}=\left[\begin{array}{cc}
D_{k-1} & 0 \\
0 & \widetilde{K}_{k}-\gamma \widetilde{M}_{k}
\end{array}\right]
$$

with $D_{k-1}=\operatorname{diag}\left(\kappa_{1}-\gamma \mu_{1}, \cdots, \kappa_{k-1}-\gamma \mu_{k-1}\right) \in \mathbb{R}^{(k-1) \times(k-1)}$.

Suppose that we have inverted the symmetric, possibly indefinite, matrix $K-\gamma M$. This can be done via a block $L D L^{T}$ factorization,

$$
\Pi(K-\gamma M) \Pi^{T}=L D L^{T}
$$

where $L$ is unit lower triangular $D$ is block diagonal with $1 \times 1$ or $2 \times 2$ diagonal blocks and $\Pi$ is a permutation matrix. If a partial diagonal pivoting strategy is used for the determination 
of $\Pi$ then the block $L D L^{T}$ factorization is numerically stable as long as the growth factor is small [7, Ch.11]. The whole inversion requires $O\left(n^{3}\right)$ operations.

At each new step of the tridiagonalization procedure, the inverse of $K_{k}-\gamma M_{k}$ is updated via

$$
\begin{aligned}
\left(K_{k}-\gamma M_{k}\right)^{-1} & =G_{k-1}^{-1}\left(K_{k-1}-\gamma M_{k-1}\right)^{-1} G_{k-1}^{-T} \\
& =\left[\begin{array}{cc}
D_{k-1}^{-1} & 0 \\
0 & \widetilde{G}_{k-1}^{-1}\left(K_{k-1}-\gamma M_{k-1}\right)^{-1} \widetilde{G}_{k-1}^{-T}
\end{array}\right]
\end{aligned}
$$

with

$$
\widetilde{G}_{k-1}^{-1}=\left[\begin{array}{cc}
1 & 0 \\
0 & H_{k-1}
\end{array}\right]\left(I-\frac{x_{k} y_{k}^{T}}{1+x_{k}^{T} y_{k}}\right) .
$$

As explained in section 2.3, the update at step $k$ can be done in $O\left((n-k+1)^{2}\right)$ operations. Therefore the computation of all the $x_{k}$ requires $O\left(n^{3}\right)$ operations.

Note that

$$
y_{k}=-\left[\left(x_{k}+e_{1}\right)^{T}\left(\gamma \widetilde{K}_{k}+\widetilde{M}_{k}\right) x_{k}\right]^{-1} V V^{T}\left(\gamma \widetilde{K}_{k}+\widetilde{M}_{k}\right)\left(x_{k}+e_{1}\right)+V w_{k}+e_{1}
$$

depends on the free vector $w_{k}$. If $w_{k}=e_{1}$ then the Householder transformation can be omitted. The complete algorithm with this particular choice for $w_{k}$ is summarized below. We assume that a function computing a block $L D L^{T}$ factorization is available. For example we can use the MATLAB function ldlt_symm from Higham's Matrix Computation Toolbox [6].

Algorithm 3.1 (Simultaneous tridiagonalization) Given two $n \times n$ symmetric matrices $K, M$ and a nonzero scalar $\gamma$ such that $K-\gamma M$ is nonsingular, the following algorithm overwrites $K$ and $M$ with the tridiagonal matrices $Q^{T} K Q$ and $Q^{T} M Q$, where the nonsingular matrix $Q$ is the product of elementary transformations of the form $I+x y^{T}$.

$$
\begin{aligned}
& Q=I \\
& {[L, D, \Pi]=\operatorname{ldlt} \operatorname{symm}_{-}(K-\gamma M)} \\
& N=\Pi^{T} L^{-T} D^{-1} L^{-1} \Pi \\
& \text { for } k=1: n-2 \\
& \quad[x, y]=\operatorname{elm}_{-} \mathrm{w}_{-} \mathrm{xy}\left(K(k: n, k: n), M(k: n, k: n), \gamma, e_{1}, N(k: n, k)\right) \\
& \quad K(k: n, k: n)=\operatorname{elm}_{-} \operatorname{apply}(K(k: n, k: n), x, y) \\
& \quad M(k: n, k: n)=\operatorname{elm}_{-} \operatorname{apply}(M(k: n, k: n), x, y) \\
& \quad N(k: n, k: n)=\operatorname{elm}_{-} \operatorname{apply}\left(N(k: n, k: n),-y /\left(1+x^{T} y\right), x\right) \\
& \quad Q(:, k: n)=Q(:, k: n)+(Q(:, k: n) x) y^{T}
\end{aligned}
$$

end

This algorithm requires about $8 n^{3}$ operations.

Note that the choice $w_{k}=e_{1}$ at each step of the reduction avoid the need to apply the Householder transformation with $H_{k}$, saving therefore some computation. However in the next section we show that this choice for $w_{k}$ may not be the best for the numerical stability of the reduction. 


\section{MINIMIZING THE CONDITION NUMBER OF $L=I+x y^{T}$}

To minimize the growth of inherent errors in the pair $(K, M)$ it is crucial to keep the condition number of the transformations used during the tridiagonalization process small. This can be explained as follows. Assume that a symmetric matrix $A$ is affected by some error $E=E^{T}$ and let $Q$ be nonsingular. We have

$$
Q^{T}(A+E) Q=\widetilde{A}+F, \quad \widetilde{A}=Q^{T} A Q, \quad F=Q^{T} E Q .
$$

To find a bound for $\|F\|$ we make use of a theorem of Ostrowski [8, p. 224]. Here the eigenvalues of a symmetric $n \times n$ matrix $N$ are ordered $\lambda_{n} \leq \cdots \leq \lambda_{1}$.

Theorem 4.1. Let $A \in \mathbb{R}^{n \times n}$ be symmetric and $Q \in \mathbb{R}^{n \times n}$ nonsingular. Then for each $k=1: n$,

$$
\lambda_{k}\left(Q^{T} E Q\right)=\theta_{k} \lambda_{k}(E),
$$

where $\lambda_{n}\left(Q^{T} Q\right) \leq \theta_{k} \leq \lambda_{1}\left(Q^{T} Q\right)$.

From this result we have

$$
\sigma_{\min }(Q)^{2}\|E\| \leq\|F\| \leq \sigma_{\max }(Q)^{2}\|E\|,
$$

where $\sigma_{\min }(Q)$ and $\sigma_{\max }(Q)$ denote the smallest and largest singular values of $Q$. Hence preand post-multiplication of a matrix $A$ by $Q$ magnifies inherent errors in $A$ by a factor at least $\sigma_{\min }(Q)^{2}$ and at most $\sigma_{\max }(Q)^{2}$. From $(25)$ we obtain

$$
\frac{\|F\|}{\|\widetilde{A}\|} \leq \kappa(Q)^{2} \frac{\|E\|}{\|A\|}, \quad \kappa(Q)=\frac{\sigma_{\max }(Q)}{\sigma_{\min }(Q)} \geq 1 .
$$

Hence the error in $A$ can be magnified by as much as $\kappa(Q)^{2}$ in passing to $\widetilde{A}$.

Recall that in our simultaneous tridiagonalization

$$
Q=G_{1} G_{2} \ldots G_{n-2}
$$

where $G_{k}=\operatorname{diag}\left(I_{k}, \widetilde{G}_{k}\right)$ with $\widetilde{G}_{k}=L_{k} H_{k}$. Householder matrices have 2-norm condition number 1 since they are orthogonal. Hence,

$$
\kappa(Q) \leq \prod_{k=1}^{n-2} \kappa_{2}\left(L_{k}\right)
$$

This suggests that in order to minimize the growth of errors we should keep $\kappa_{2}\left(L_{k}\right)$ small at each step of the tridiagonalization procedure. In the rest of this section, we show how to choose the vector $w$ in $(23)$ so that $\kappa_{2}(L)$ is minimized.

By definition,

$$
\kappa(L)=\frac{\sigma_{\max }(L)}{\sigma_{\min }(L)}=\left(\frac{\lambda_{\max }\left(L^{T} L\right)}{\lambda_{\min }\left(L^{T} L\right)}\right)^{1 / 2} .
$$

Let $y_{k}, k=1: \ell-2$ be $\ell-2$ linearly independent vectors orthogonal to $[x, y]$. Then

$$
L^{T} L y_{k}=\left(I+y x^{T}\right)\left(I+x y^{T}\right) y_{k}=y_{k},
$$


which implies that $L^{T} L$ has $\ell-2$ eigenvalues equal to 1 . We denote by $\lambda_{1}, \lambda_{2}$ the remaining two eigenvalues. We have

$$
\lambda_{1} \lambda_{2}=\operatorname{det}\left(L^{T} L\right)=\operatorname{det}(L)^{2}=\left(1+y^{T} x\right)^{2}
$$

and

$$
\begin{aligned}
(\ell-2)+\lambda_{1}+\lambda_{2} & =\operatorname{trace}\left(L^{T} L\right) \\
& =\operatorname{trace}\left(I+x y^{T}+y x^{T}+\left(x^{T} x\right) y y^{T}\right) \\
& =\ell+2 y^{T} x+\|x\|^{2}\|y\|^{2} .
\end{aligned}
$$

Let

$$
p=2\left(1+y^{T} x\right), \quad q=\|x\|^{2}\|y\|^{2} .
$$

Then $\lambda_{1}$ and $\lambda_{2}$ are the roots of

$$
\lambda^{2}-(p+q) \lambda+\frac{p^{2}}{4}=0
$$

and hence

$$
\kappa(L)=\left(\frac{p+q+\sqrt{q(q+2 p)}}{p+q-\sqrt{q(q+2 p)}}\right)^{1 / 2} .
$$

Defining

$$
r(y) \equiv \frac{q}{p}=\frac{\|x\|^{2}\|y\|^{2}}{2\left(1+y^{T} x\right)}
$$

we have

$$
\min _{y \in \mathbb{R}^{\ell}} \kappa(L)=\min _{y \in \mathbb{R}^{\ell}} \frac{1+r(y)+\sqrt{r(y)(r(y)+2)}}{1+r(y)-\sqrt{r(y)(r(y)+2)}},
$$

and the optimal $y$ minimizes

$$
\min _{y \in \mathbb{R}^{\ell}} \sqrt{r(y)(r(y)+2)}
$$

and hence also

$$
\min _{y \in \mathbb{R}^{\ell}} r(y) .
$$

We first show that $r(y)$ is minimized for $y \in \operatorname{span}\left(x, e_{1}\right)$, that is, $y=\alpha x+e_{1}$ for some $\alpha \in \mathbb{R}$ (recall that $e_{1}^{T} x=0$ and $e_{1}^{T} y=1$ ) and then determine the scalar $\alpha$. Let $y_{1}=y+z$ with $z \in \operatorname{span}\left(x, e_{1}\right)^{\perp}$. We have

$$
p_{1} \equiv 2\left(1+y_{1}^{T} x\right)=p, \quad q_{1} \equiv\|x\|^{2}\left\|y_{1}\right\|^{2}=\|x\|^{2}\left(1+\|x\|^{2}\right)>p
$$

so that $r(y)<r\left(y_{1}\right)$. Then it is easy to show that the function

$$
g(\alpha) \equiv r(y)=\|x\|^{2}\left(1+\alpha^{2}\|x\|^{2}\right) /\left(2\left(1+\alpha\|x\|^{2}\right)\right)
$$

is minimized for

$$
\alpha=-\frac{1+\sqrt{1+\|x\|^{2}}}{\|x\|^{2}}
$$


yielding

$$
y=e_{1}-\frac{1+\sqrt{1+\|x\|^{2}}}{\|x\|^{2}} x
$$

and

$$
w=V^{T} y+\left[\left(x+e_{1}\right)^{T}(\gamma K+M) x\right]^{-1} V^{T}(\gamma K+M)\left(x+e_{1}\right) .
$$

Note that with this expression for $y$, we have $x^{T} y \neq-1$ so that $L$ is nonsingular.

The optimal condition number for $L$ is given by

$$
\kappa_{\text {opt }}(L)=\frac{\sqrt{1+\|x\|^{2}}+\|x\|}{\sqrt{1+\|x\|^{2}}-\|x\|}
$$

and is reasonably small as long as $\|x\|=O(1)$.

Given $z=(K-\gamma M)^{-1} e_{1}$, the computation of $x$ and $y$ requires $9 \ell$ operations and can be written in pseudocode as follows.

$$
\begin{aligned}
& \text { function }[x, y]=\operatorname{elm}_{-} \mathrm{xy}(K, M, z) \\
& \% \text { Construct the vectors } x, y \in \mathbb{R}^{\ell} \text { so that } L=I+x y^{T} \text { satisfies (11) with } w \text { given by (29). } \\
& \begin{array}{l}
k=K(2: \ell, 1), m=M(2: \ell, 1) \\
\text { If }\left|k^{T} m\right|=\|k\|_{2}\|m\|_{2}
\end{array} \\
& \quad \begin{array}{l}
\quad x=0, y=0 \\
\text { else } \quad \\
\qquad \begin{array}{l}
x=z / z(1), x(1)=0 \\
\text { end }
\end{array}
\end{array}
\end{aligned}
$$

This yields the following algorithm.

Algorithm 4.2 (Simultaneous tridiagonalization) Given two $n \times n$ symmetric matrices $K, M$ and a nonzero scalar $\gamma$ such that $K-\gamma M$ is nonsingular, the following algorithm overwrites $K$ and $M$ with the tridiagonal matrices $Q^{T} K Q$ and $Q^{T} M Q$, where the nonsingular matrix $Q$ is the product of Householder matrices and elementary matrices of the form $I+x y^{T}$ having minimal condition number.

$$
\begin{aligned}
& Q=I \\
& {[L, D, \Pi]=\operatorname{ldlt} \_\operatorname{symm}(K-\gamma M)} \\
& N=\Pi^{T} L^{-T} D^{-1} L^{-1} \Pi \\
& \text { for } k=1: n-2 \\
& \quad[x, y]=\operatorname{elm} \_x y(K(k: n, k: n), M(k: n, k: n), N(k: n, k)) \\
& \quad K(k: n, k: n)=\operatorname{elm} \_\operatorname{apply}(K(k: n, k: n), x, y) \\
& \quad M(k: n, k: n)=\text { elm_apply }(M(k: n, k: n), x, y) \\
& \quad N(k: n, k: n)=\operatorname{elm} \text { apply }\left(N(k: n, k: n),-y /\left(1+x^{T} y\right), x\right) \\
& \quad Q(:, k: n)=Q(:, k: n)+(Q(:, k: n) x) y^{T} \\
& \quad v=\text { house }(K(k+2: n, k+1)) \\
& \quad x_{1}=\left[0,-2 v^{T} /\left(v^{T} v\right)\right]^{T}, y_{1}=\left[0, v^{T}\right]^{T} \\
& \quad K(k: n, k: n)=\operatorname{elm}_{-} \operatorname{apply}\left(K(k: n, k: n), x_{1}, y_{1}\right)
\end{aligned}
$$




$$
\begin{array}{ll} 
& M(k: n, k: n)=\operatorname{elm}_{-} \operatorname{apply}\left(M(k: n, k: n), x_{1}, y_{1}\right) \\
& N(k: n, k: n)=\operatorname{elm}_{-} \operatorname{apply}\left(N(k: n, k: n), x_{1}, y_{1}\right) \\
& \left.Q(:, k: n)=Q(:, k: n)+Q(:, k: n) x_{1}\right) y_{1}^{T} \\
\text { end } &
\end{array}
$$

This algorithm requires about $13 n^{3}$ operations which is about $50 \%$ more than Algorithm 3.1.

\section{COMMENTS ON HOW TO CHOOSE $\gamma$}

A bad choice for $\gamma \neq 0$ may affect the numerical stability of the reduction. Ideally, we would like to choose $\gamma$ to minimize

$$
\kappa(K-\gamma M)=\frac{\max \left|\lambda_{j}(\gamma)\right|}{\min \left|\lambda_{j}(\gamma)\right|}
$$

where the $\lambda_{j}(\gamma)$ are the eigenvalues of $K-\gamma M$. This is a difficult nonlinear optimization problem.

If one knows about the location of the eigenvalues of the pair $(K, M)$ then one can choose $\gamma$ to be outside the region where the eigenvalues lie. For example if $(K, M)$ comes from a first order system which is known to be stable then all the eigenvalues lie in the left half plane and it is advisable to take $\gamma>0$.

If no special information is known about the problem, we suggest to take

$$
\gamma= \pm \frac{\|K\|_{1}}{\|M\|_{1}}
$$

where $\|\cdot\|_{1}$ denotes the matrix 1-norm, and to choose the sign for $\gamma$ that maximizes $\|K-\gamma M\|_{1}$. This choice of sign minimizes the risk of cancellation in computing $K-\gamma M$ but does not guarantee that the resulting $\gamma$ is far from an eigenvalue.

A simple test to check for a "bad $\gamma$ " is to compute the condition number of the block diagonal matrix $D$ in the $L D L^{T}$ factorization of $K-\gamma M$. If $\kappa(D)$ is large then one can choose another value of $\gamma$, compute the $L D L^{T}$ factorization of the new $K-\gamma M$ and check $\kappa(D)$ again. It is unlikely this process will need to be repeated many times. The $L D L^{T}$ factorization costs about $n^{3} / 3$ operations, which is small compared with the total number of operations needed for the complete simultaneous tridiagonalization process. Recall that Algorithm 3.1 requires about $8 n^{3}$ operations and Algorithm 4.2 requires about $13 n^{3}$ operations. This is an example of a tradeoff between numerical stability and computational cost.

\section{NUMERICAL EXPERIMENTS}

Our aim in this section is to investigate the numerical properties of the simultaneous tridiagonalization procedures just described. In our tests following quantities are computed:

- the two scaled residual errors

$$
\mathcal{R}_{K}=\frac{\left\|Q^{T} K Q-T\right\|}{\|K\|\|Q\|^{2}}, \quad \mathcal{R}_{M}=\frac{\left\|Q^{T} M Q-S\right\|}{\|M\|\|Q\|^{2}},
$$


Figure 1. Residuals and condition numbers for 20 random matrices. Results from trd_L are marked with ' $x$ ' and results from trd_LH are marked with 'o'.

- the condition number $\kappa_{2}(Q)$ of the transformation $Q$,

- the largest condition number $\kappa(G)=\max _{1 \leq k \leq n-2} \kappa\left(G_{k}\right)$ of the individual transformations $G_{k}$ used during the tridiagonalization process,

All our tests have been performed with MATLAB. We named our MATLAB implementations

- $\operatorname{trd}$ _L: tridiagonalization by non-orthogonal rank-one modifications of the identity matrix (Algorithm 3.1).

- trd_LH: tridiagonalization by mixed Householder reflectors and non-orthogonal rank-one modifications of the identity matrix with minimal condition numbers (Algorithm 4.2).

We ran a set of tests with random matrices of the form

$$
\mathrm{K}=\operatorname{randn}(\mathrm{n}) ; \mathrm{K}=\mathrm{K}+\mathrm{K}^{\prime} ; \mathrm{M}=\operatorname{randn}(\mathrm{n}) ; \mathrm{M}=\mathrm{M}+\mathrm{M}^{\prime} \text {. }
$$

We took $n=50$ and chose $\gamma$ as in (30). The residuals $\mathcal{R}_{M}$ and $\mathcal{R}_{K}$ in (31) and the corresponding condition numbers $\kappa(Q)$ and $\kappa(G)$ are plotted in Figure 1 for 20 random matrices. The lines with ' $x$ ' corresponds to residuals obtained with trd_L and the lines with 'o' corresponds to residuals obtained with trd_LH. Clearly, the reduction using a combination of Householder reflectors and rank-one modifications of the identity matrix with minimal condition numbers yields smaller residuals and condition numbers. The residuals $\mathcal{R}_{M}$ and $\mathcal{R}_{K}$ corresponding to trd_L all lie between $10^{-6}$ and $10^{-12}$ and are on average of the order $10^{-7}$. For trd_LH the residuals $\mathcal{R}_{M}$ and $\mathcal{R}_{K}$ lie between $10^{-13}$ and $10^{-16}$ and are on average of the order $10^{-14}$. This shows a large improvement of the quality of the results over those obtained with trd_L. Note that for this set of random problems and for a particular algorithm, $\mathcal{R}_{M}$ and $\mathcal{R}_{K}$ have the same magnitude. On average, $\kappa(Q)$ is of the order $10^{5}$ if $Q$ if computed with trd_L and of the order $10^{3}$ if computed from trd_LH. The last plot in Figure 1 shows that a large condition number for one of the $G_{k}$ directly affects the residuals and confirms the fact that it is crucial to keep the condition number of each transformation small.

We ran similar tests with random singular matrices $M$ and tests with positive definite $K$ and $M$ and obtained similar conclusions: trd_LH behaves more stably than trd_L.

We studied the numerical behavior of trd_LH with respect to $\gamma$ on several random test matrices by letting $\gamma$ vary between the smallest and largest real eigenvalues of $(K, M)$. The plots in Figure 2 show $\kappa(K-\gamma M), \mathcal{R}_{\max }=\max \left(\mathcal{R}_{K}, \mathcal{R}_{M}\right)$ and $\kappa(Q)$ for a $6 \times 6$ random pair $(K, M)$ and values of $\gamma$ between -2 and 2.5. The real eigenvalues of $(K, M)$ are marked with ' $*$ ' on the $\gamma$-abscisse. As expected, values of $\gamma$ in the neighborhood of these real eigenvalues yields large values for $\kappa(K-\gamma M)$. This directly affects the quality of the computed tridiagonal matrices but does not seem to interfere with the value of $\kappa(Q)$. 
Figure 2. Influence of $\gamma$ on $\kappa(K-\gamma M), \mathcal{R}_{\max }=\max \left(\mathcal{R}_{K}, \mathcal{R}_{M}\right)$ and $\kappa(Q)$ for a $6 \times 6$ random pair of symmetric matrices $(K, M)$.

\section{CONCLUSION}

A new method has been presented whereby a pair of symmetric matrices $(K, M)$ is successively transformed using elementary transformations (rank-one modifications of the identity matrix) in such a way that the resulting two matrices $(T, S)$ are both tridiagonal. The total number of operations needed in this reduction process is $O\left(n^{3}\right)$. No assumption on the positivity or nonsingularity of $K$ and $M$ is required. However it is important to choose $\gamma$ so that $K-\gamma M$ is well-conditioned.

We provide two algorithms: Algorithm 3.1, which uses only non-orthogonal elementary transformations and Algorithm 4.2, more costly but with better numerical stability properties, which uses a combination of orthogonal transformations and non-orthogonal elementary transformations with minimal condition numbers. All our numerical experiments confirm the numerical superiority of Algorithm 4.2.

Our simultaneous tridiagonalizing process has particular relevance to the quadratic eigenvalue problem

$$
\left(\lambda^{2} M+\lambda D+K\right) x=0,
$$

for two reasons. Firstly, symmetric linearizations of that problem usually result in a generalized eigenvalue problem in which neither matrix is positive definite [9]. More importantly, however, this process may serve as a prototype for an analogous procedure for the quadratic eigenvalue problem in which all three symmetric matrices are modified in steps using elementary structurepreserving coordinate transformations [4] such that the eigenvalues of the system are preserved but the structure of all three system matrices becomes progressively more tridiagonal.

\section{ACKNOWLEDGEMENTS}

The authors acknowledge the funding of the Engineering and Physical Sciences Research Council (EPSRC) through the two linked grants GR/M93062 and GR/M93079, entitled 'The Application of Geometric Algebra to Second Order Dynamic Systems in Engineering' from which this work derives. Friswell gratefully acknowledges the support of the EPSRC through the award of an Advanced Fellowship.

Tisseur is grateful for the support of EPSRC, for this work, thought the grant number GR/R45079 and for the support of the Nuffield Foundation through grant number NAL/00216/G.

\section{REFERENCES}

1. M. A. Brebner and J. Grad, Eigenvalues of $A x=\lambda B x$ for real symmetric matrices $A$ and $B$ computed by reduction to a pseudosymmetric form and the HR process, Linear Algebra and Appl., 43 (1982), pp. 99118.

2. Angelika Bunse-Gerstner, An algorithm for the symmetric generalized eigenvalue problem, Linear Algebra and Appl., 58 (1984), pp. 43-68.

3. Seamus D. Garvey, Michael I. Friswell, and Uwe Prells, Coordinate transformations for general second-order systems, part I: General transformations. Manuscript, 2001. To appear in J. Sound Vibration. 
4. - Coordinate transformations for general second-order systems, part II: Elementary structure preserving transformations. Manuscript, 2001. To appear in J. Sound Vibration.

5. Gene H. Golub and Charles F. Van Loan, Matrix Computations, Johns Hopkins University Press, Baltimore, MD, USA, third ed., 1996.

6. Nicholas J. Higham, The matrix computation toolbox. http://www.ma.man.ac.uk/^higham/mctoolbox.

7. — Accuracy and Stability of Numerical Algorithms, Society for Industrial and Applied Mathematics, Philadelphia, PA, USA, second ed., 2002.

8. Roger A. Horn and Charles R. Johnson, Matrix Analysis, Cambridge University Press, 1985.

9. Françoise Tisseur and Karl Meerbergen, The quadratic eigenvalue problem, SIAM Review, 43 (2001), pp. $235-286$.

10. Rudolf Zurmühl ANd Sigurd FAlK, Matrizen und ihre Anwendungen für angewandte Mathematiker, Physiker und Ingenieure. Teil 2, Springer-Verlag, Berlin, Berlin, fifth ed., 1986. 